\title{
Contribution des savoir-faire viticoles au développement durable et à la démarche de responsabilité Sociétale et environnementale, dans le cadre d'une gouvernance patrimoniale des terroirs / Contribution of wine know-how to the sustainable development and the approach of social responsibility and environmental, within the framework of a patrimonial governance of the terroirs
}

Joël Rochard

IFV (Institut Français de la Vigne et du Vin), 17 rue Jean Chandon Moët, 51200 Epernay, France

\begin{abstract}
Résumé. Les savoirs viticoles constituent, au-delà des atouts locaux, un patrimoine humain irremplaçable. Le génie des hommes a permis à de nombreux écosystèmes terrestres, y compris parfois les plus extrêmes, d'être valorisés afin d'exprimer la quintessence du vin, expression des terroirs, ainsi que des particularités historiques et culturelles locales. Au-delà de la dimension territoriale, les savoir-faire viticoles sont également porteurs de dynamique locale, de préservation et de valorisation. Par ailleurs, ceux-ci peuvent alimenter la créativité scientifique et technologique dans une période de post modernité, en proie à des doutes et parfois des impasses, en quête de valeurs traditionnelles. La communication, à partir d'exemples, souligne la contribution des savoirs historiques locaux à une culture et une gouvernance, indispensable à la valorisation des terroirs et des vins, mais également au développement de démarche opérationnelle du développement durable en lien notamment avec la responsabilité sociale et environnementale de la filière viticole, vis-à-vis de notre planète et des générations futures.
\end{abstract}

\begin{abstract}
The wine knowledge constitutes, beyond the local assets, an irreplaceable human inheritance. Human engineering allowed many terrestrial ecosystems, including sometimes most extreme, to be developed in order to express the quintessence of the wine, expression of the terroirs, as well as historical and cultural characteristics local. Beyond territorial dimension, wine know-how is also carrying local dynamics, safeguarding and valorization. In addition, those can feed the scientific and technological creativity during one time of post modernity, in the grip of doubts and sometimes of the dead ends, in search of traditional values. The communication, starting from examples, underlines the contribution of the local historical knowledge to a culture and a governance, essential to the valorization of the terroir and wines, but also to the development of approach operational of sustainable development in link in particular with the social responsibility and environmental of the wine sector, with respect to our planet and of the future generations.
\end{abstract}

\section{Introduction}

La fin des années 1970 et le début des années 1980 marquent la fin de la période des «30 glorieuses», caractérisée par l'intensification des méthodes de production. Elle a permis de réduire la pénibilité des travaux agricoles et viticoles, de régulariser les rendements et de proposer aux consommateurs des denrées abondantes, variées et bon marché. Mais si elle a apporté une réponse à la fois sociale et économique au problème posé par la population dans un contexte de sortie guerre, cette mutation s'est accompagnée dans le même temps de multiples effets collatéraux sur l'environnement. Dès les années 1970, l'absence de prise en compte des équilibres naturels, couplée à la première crise pétrolière, déclencha l'émergence de nouveaux courants de pensée écologiste. En 1972, le rapport Meadows commandé par le Club de Rome, «Les limites de la croissance», prévoyait un effondrement brutal des économies au cours du $21^{\mathrm{e}}$ siècle suite aux effets conjugués de l'évolution démographique, des pressions environnementales et de l'épuisement des ressources.

Depuis la Conférence des Nations Unies de Stockholm en 1972, le problème du rapport entre le développement économique et la détérioration de l'environnement s'est inscrit au centre des préoccupations internationales.

En 1983, L'ONU créa la Commission Mondiale pour l'Environnement et le Développement qui élabora le concept de développement durable : «Répondre aux besoins du présent sans compromettre la capacité des générations futures de satisfaire leurs propres besoins». En 1992, 172 états se sont réunis à la conférence des Nation Unies à Rio, au cours de laquelle, cinq accords importants furent adoptés, visant à infléchir l'orientation expansionniste du développement (Fig. 1). 


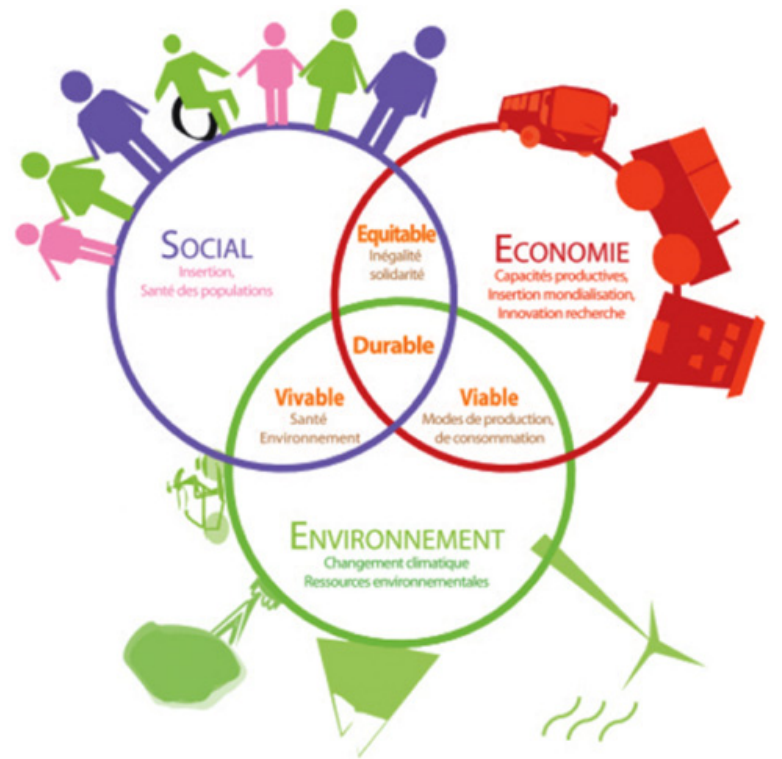

Figure 1. Les trois piliers du développement durable (développement durable source: www.ledepartement66.fr).

\section{Emergence de la responsabilité sociétale en lien avec l'environnement}

La notion de développement durable a mis l'accent sur l'impact de l'homme, par son activité domestique, agricole ou industrielle, sur le devenir des générations futures. Ainsi, la biodiversité, l'utilisation des énergies fossiles, la production de déchet et de gaz à effet de serre, s'immiscent progressivement dans les itinéraires viticoles. Parallèlement la crédibilité des démarches s'appuie de plus en plus sur des notions d'indicateurs en lien avec des méthodologies de type Analyse de Cycle de Vie (Fig. 2).

$\mathrm{Au}$-delà des aspects environnementaux, un peu plus tardivement, une approche sociale puis sociétale du développement durable s'est également intégrée dans la réflexion stratégique de la filière viticole et a été formalisé en France par l'application viticole la norme ISO 26000.

«L'inégalité a un prix, elle est la cause et la conséquence de la faillite du système politique et elle alimente, dans notre système économique, une instabilité et une inefficacité qui l'aggravent à leur tour. C'est ce cercle vicieux qui nous plonge dans l'abîme. (..) Puisqu'il est flagrant que notre système économique ne peut rien pour la plupart des citoyens et que nos gouvernements sont globalement sous la coupe des intérêts privés, la confiance dans la démocratie va s'éroder. Et puisque nous comprenons peu à peu que nombre de nos pays ne sont plus ceux de l'égalité des chances et du fair play, c'est sans doute notre sentiment de la justice qui est menacé.» Joseph E. Stieglitz, Le prix de l'inégalité.

Le concept de RSE n'apparait qu'à partir des années 1960 dans la littérature consacrée aux entreprises. Depuis les années 1980, sous l'impulsion notamment des ONG, les concepts de finance éthique, commerce équitable et développement durable sont entrés dans le débat des instances politiques. Des recherches sur la responsabilité sociale et environnementale se sont concrétisées par la mise au point de référentiels internationaux, des guides de conduite des entreprises ou des certificats, normes,

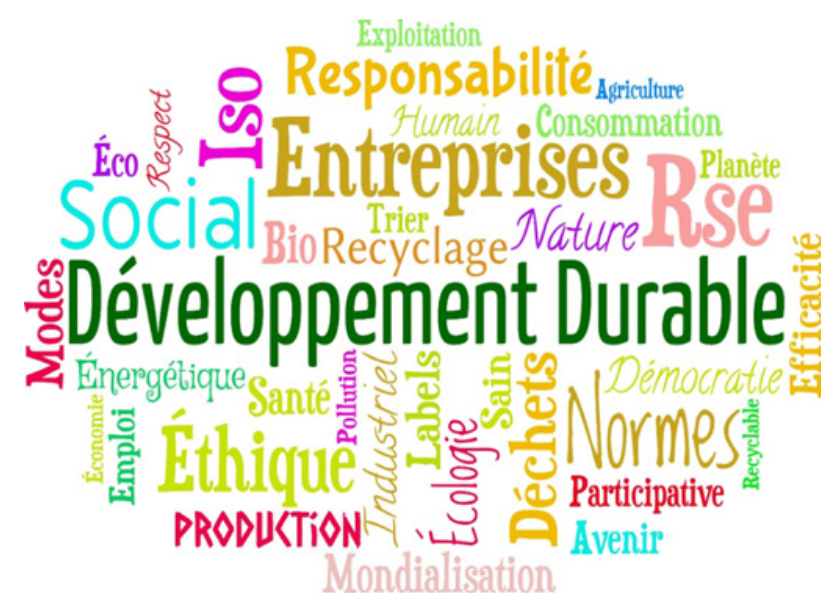

Figure 2. Le développement durable, une approche intégrative. (Source www.ocealia-groupe.fr).

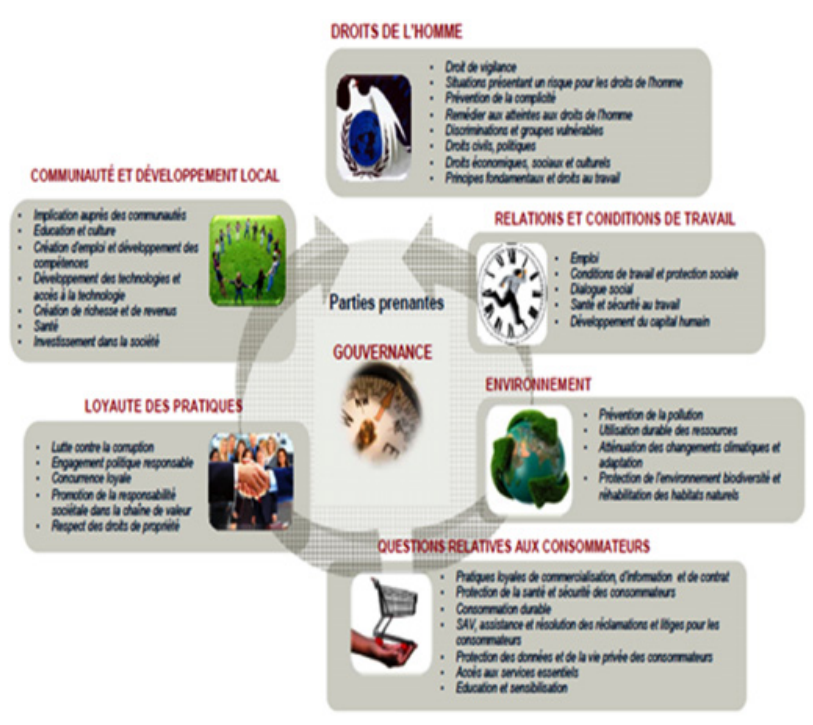

Figure 3. Les 7 points centraux de la démarche RSE/ISO 26000 (Source www.luxor-lighting.com).

label validé par des audits sociaux et environnementaux. C'est dans ce contexte qu'a été formalisée la norme internationale ISO 26000 (lignes directrices relatives à la responsabilité sociétale).

Pour la Commission européenne, la RSE est un «concept dans lequel les entreprises intègrent les préoccupations sociales, environnementales et économiques dans leurs activités et dans leurs interactions avec leurs parties prenantes sur une base volontaire».

La responsabilité sociétale et environnementale vise en premier lieu les besoins élémentaires des hommes: conditions de travail, nourriture, santé, bien-être, etc. Au-delà, une démarche RSE suppose également une implication de l'entreprise ou de l'organisation vis-àvis des communautés locales mais également à l'échelle planétaire, en lien notamment avec l'éducation et la culture. Cette vision intègre en particulier la préservation et la valorisation d'un patrimoine commun issu le plus souvent de l'organisation d'une communauté et de la diffusion au cours du temps d'un savoir-faire collectif, autant de sujet qui sont ancrés dans l'histoire de la vigne et du vin. 


\begin{abstract}
Responsabilité sociétale de la filière vin : un guide AFNOR à la disposition de tous les acteurs

Le guide AFNOR, réalisé par une quarantaine de professionnels de la filière vin notamment, aborde tous les aspects de la profession. Impulsé Y. Chabin au sein de l'Interprofession des Vins Pays d'Oc IGP, il a été élaboré collectivement, sous l'égide d'AFNOR, par une quarantaine d'organismes professionnels.

Le guide (AC X30-032), d'application volontaire, dresse une liste de recommandations pour les entreprises souhaitant initier ou améliorer leur stratégie et leurs actions en matière de RSE. Il concrétise les enjeux de la responsabilité sociétale pour la filière vin en s'appuyant sur le document le plus légitime sur ce sujet: la norme internationale ISO 26000 ainsi que les documents et les retours d'expérience associés.

Des préconisations concrètes et opérationnelles sont proposées pour intégrer la RSE dans le projet d'entreprise. Sur le volet social par exemple, les enjeux des conditions de travail sont traités précisément pour favoriser le développement du capital humain. Pour ceux de la sous-traitance, les propositions ont pour objectif d'avoir des pratiques loyales et respectueuses. Sur le volet environnemental, tous les professionnels engagés ont notamment défini des repères pour prévenir les pollutions, utiliser les ressources durablement et maintenir la biodiversité. Le lien est aussi fait avec la protection et l'information du consommateur sur les produits et les métiers de la filière (Fig. 4).
\end{abstract}

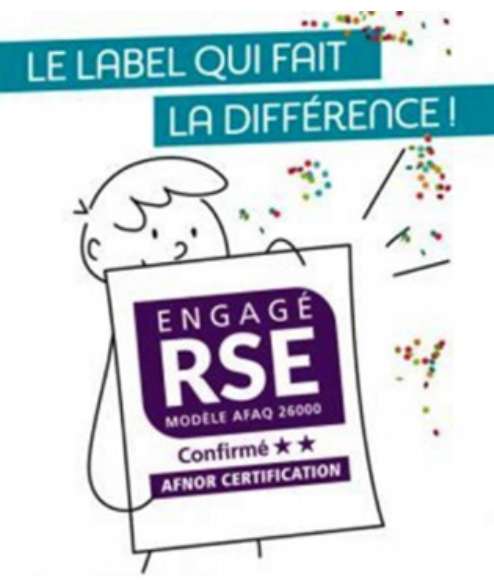

Figure 4. Accompagnement des démarches RSE par l'AFNOR http://www . afnor .org/actualites/responsabilitesocietale-de-la-filiere-vin-guide/.

Les savoirs viticoles constituent, au-delà des atouts locaux, un patrimoine humain irremplaçable. Le génie humain a permis à de nombreux écosystèmes terrestres, y compris parfois les plus extrêmes, d'être valorisés afin d'exprimer la quintessence du vin, expression des terroirs, ainsi que des particularités historiques et culturelles locales.

Depuis l'invention de l'agriculture au cours du néolithique, il y a 10000 ans au Moyen-Orient, l'homme a su s'adapter à l'essor démographique par des savoirfaire initialement empiriques, mais validés au fil des générations. Très tôt les échanges entre les peuples ont permis une confrontation des savoirs et ont contribué

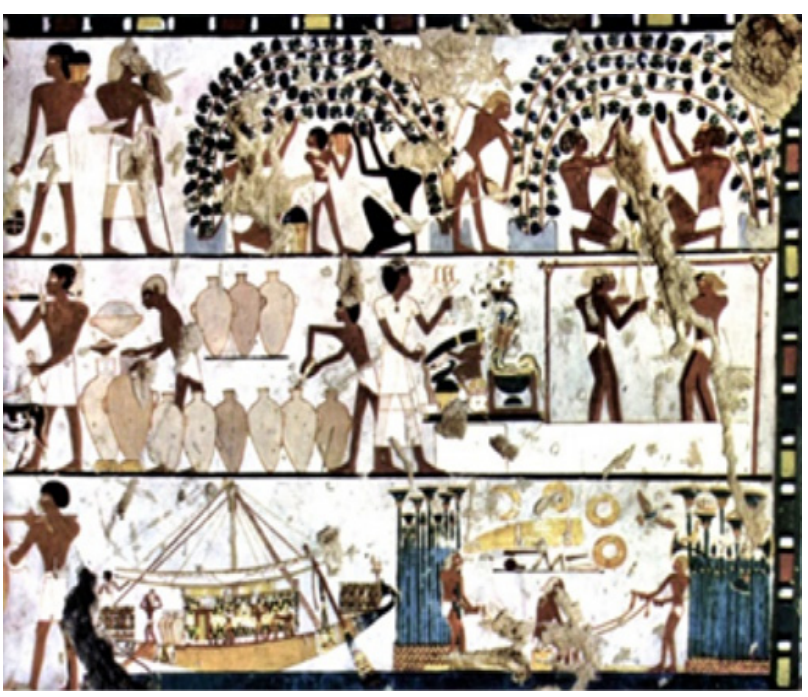

Figure 5. Fresque égyptiennes de la tombe de Khaemouaset, grand-prêtre sous le règne d'Amenhotep 1 vers - 1500 avant J.C., qui témoigne du savoir-faire des Égyptiens depuis la vendange jusqu'à la mise en amphores et au transport.

à dynamiser la créativité locale. Grâce notamment aux civilisations égyptiennes, helléniques et romaines, relayées au moyen âge par les monastères, chaque région viticole européenne a su trouver des formes singulières de culture de la vigne et d'élaboration des vins, adaptées au contexte physique et historique local. Ce savoir-faire s'est transmis par la migration des hommes et des idées au pays du Nouveau Monde, avec des idées nouvelles, qui a développ une viticulture à la fois moderne mais également fortement imprégnée des savoir-faire ancestraux (Fig. 5).

Au-delà des enjeux patrimoniaux, ces savoirs sont souvent porteurs d'une identité locale, de nature à fédérer les énergies collectives autour de valeurs culturelles, indispensable à la valorisation qualitative des vins, mais également au développement opérationnel de projets durables. Bien évidemment, la protection et la valorisation des paysages s'intègrent dans cette vision patrimoniale, mais également écologique des terroirs. Le classement d'une quinzaine de sites viticoles au sein du patrimoine mondial Unesco culturels ou/et naturels et la labellisation paysagère de nombreuses autres régions, témoignent de la gouvernance locale et régionale autour des paysages. Parallèlement aux aspects strictement patrimoniaux, le paysage, dans une stratégie collective d'organisation de l'espace, répond à des enjeux environnementaux notamment pour limiter les risques de ruissellement et d'érosion pour les vignobles de coteaux Il est également est au cœur de l'agroécologie qui vise à valoriser la biodiversité locale pour limiter les parasites.

\section{Préservations et valorisation des savoir-faire : de la réflexion à l'action}

La modernité, qui s'est imposé notamment après la seconde guerre mondiale, a permis parallèlement à l'autosuffisance alimentaire, de limiter la pénibilité du travail dans les exploitations agricoles et viticoles. En contrepartie, elle a souvent occulté les savoir-faire ancestraux, considérés comme inutiles ou parfois contraignant visà-vis d'une pensée «chimique ou technologiste». Ainsi 
l'histoire de l'humanité, associée au développement des sciences, s'inscrivait jusqu'à présent dans un contexte expansionniste. Toute avancée scientifique offrait de nouvelles perspectives de développement, qui semblaient sans limite. Force est de constater maintenant que les capacités d'adaptation de notre planète ne sont pas infinies.

Ainsi depuis la fin du $\mathrm{XX}^{\mathrm{e}}$ siècle, la période de postmodernité est en proie à des doutes et parfois des impasses, avec l'émergence d'un passéisme, valeur refuge vis-à-vis d'un avenir incertain. Au-delà des impacts locaux, nos terroirs et chacun d'entre nous, seront probablement demain soumis à un effet de boomerang planétaire, directement lié à l'effet cumulatif de nos comportements individuels. Le développement sans limites des technologies est remis en cause, ce qui suppose une mixité entre des innovations contextualisée par rapport à leurs effets locaux et planétaires sur le long terme et une tradition porteuse de savoir-faire ancestraux souvent validés par le temps. Par ailleurs, cette modernité, porteuse de valeurs «d'individus roi, égocentriques», relayées par le marketing et les médias, s'est également traduite par l'émergence d'un individualisme, destructeur des pratiques collectives, ce qui suppose de créer à nouveau du lien entre les acteurs au sein des territoires viticoles. Fort heureusement, la filière viticole, par son attachement culturel et historique au terroir et aux valeurs ancestrales, a su conserver des savoir-faire et traditions qu'il convient de préserver et de valoriser notamment dans le cadre d'une vision de Responsabilité Sociétale et Environnementale, vis-à-vis des acteurs locaux mais plus globalement de l'ensemble de l'humanité, à l'image du classement d'une quinzaine de sites viticoles au titre du patrimoine mondial Unesco.

«L'Occident a longtemps cru que la modernité était le triomphe de la raison, la destruction des traditions, des appartenances, des croyances, la colonisation du vécu sur le calcul. Mais, aujourd'hui, toutes les catégories qui avaient été soumises à l'élite éclairée, travailleurs et colonisés, femmes et enfants, se sont révoltées et refusent d'appeler moderne un monde qui ne reconnaît pas à la fois leur expérience particulière et leurs accès à l'universel. De sorte que ceux qui s'identifient à la raison apparaissent désormais comme les défenseurs d'un pouvoir.» Alain TOURAINE, Critique de la modernité, 1992.

\subsection{Festivités autour du vin : Exemple de la Fête des vignerons de Vevey en Suisse}

Créer du lien entre les acteurs des territoires viticoles participe à une réflexion voir une dynamique collective autour du patrimoine commun, mais également dans la valorisation économique et progressivement écologique des terroirs. La fête de la Saint-Vincent dans les régions septentrionales françaises, l'élection de la «Reine des Vins» en Alsace, et dans les zones germaniques, l'organisation de festivités en lien avec l'œnotourisme, contribuent à dynamiser la vie collective dans les zones viticoles.

La Fête des vignerons de Vevey a été inscrite en 2016 sur la Liste représentative du patrimoine culturel immatériel de l'humanité. À Vevey, il existe une tradition qui célèbre le travail des vignerons de la communauté et à laquelle participent non seulement des professionnels mais aussi des membres du public et des artistes locaux.

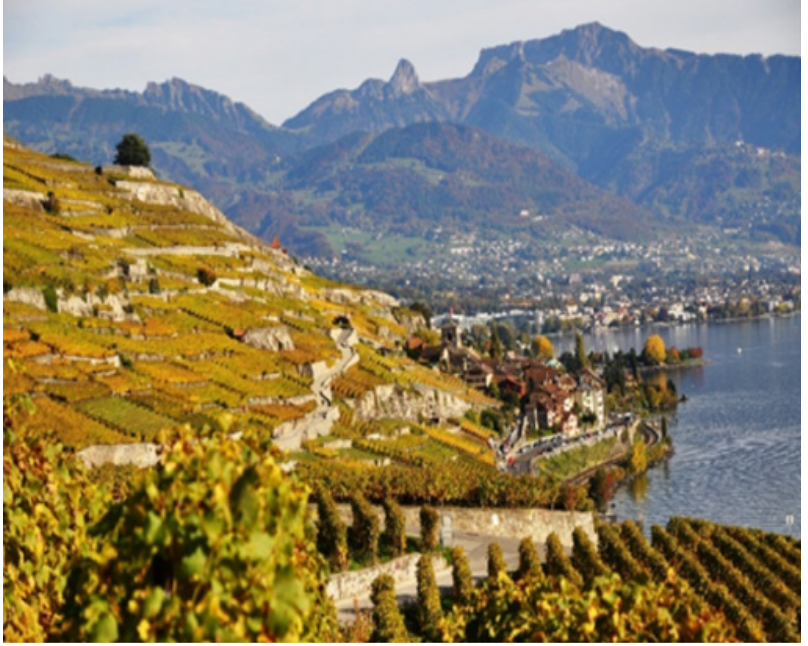

Figure 6. Paysages suisse de Vevey en bordure du lac Léman, dont la fête des vignerons a été classée par l'UNESCO sur la liste du patrimoine immatériel de l'humanité.

D'abord simple parade, la Fête des Vignerons compte aujourd'hui 15 représentations sur trois semaines et plus de 5000 figurants. Organisée tous les vingt ans, chaque fête repose sur la recréation de thèmes traditionnels tels que le travail de la terre et des vignes, le cycle des saisons et la fraternité. Des cortèges ont lieu pendant toute la durée de la fête, ainsi que des remises de prix aux meilleurs vignerons. La fête comporte aussi de la musique et des chansons, comme le Ranz des vaches (chant traditionnel des gardiens de troupeaux alpins), et des processions jusqu'à la ville voisine de La Tourde-Peilz. On peut également y rencontrer des figurants déguisés et des marmousets (figurines de bois fixées sur des bâtons). La Fête des Vignerons repose essentiellement sur le travail de bénévoles, qui la préparent pendant plusieurs années. La transmission se fait par le biais des familles et de l'association à but non lucratif de la Confrérie des Vignerons de Vevey, qui coordonne la fête et dont les membres sont issus du grand public. Cette tradition encourage l'esprit de communauté, contribue à la vie artistique et procure un sentiment de continuité culturelle, tout en stimulant le savoir-faire des artisans vignerons (Fig. 6).

\subsection{Terroir et savoir-faire monastique : exemple des climats de Bourgogne}

L'écosystème culturel que constitue un terroir dépasse largement sa dimension physique. Le vigneron est l'une des composantes essentielles. Le savoir-faire acquis de génération en génération, l'aptitude à surmonter les défis techniques, la dynamique individuelle et collective, mais également la capacité de construire et pérenniser une image ainsi que la volonté de s'impliquer dans une démarche durable sont, parallèlement à l'approche spatiale, les clés de la valorisation du produit et de la transmission aux générations futures d'un outil de production viable. A l'image de l'homme dont le prédéterminisme génétique est évident, mais pour lequel la réussite personnelle et professionnelle est largement façonnée par ses influences culturelles, le vin est le fruit d'une symbiose intime entre nature et domestication. 


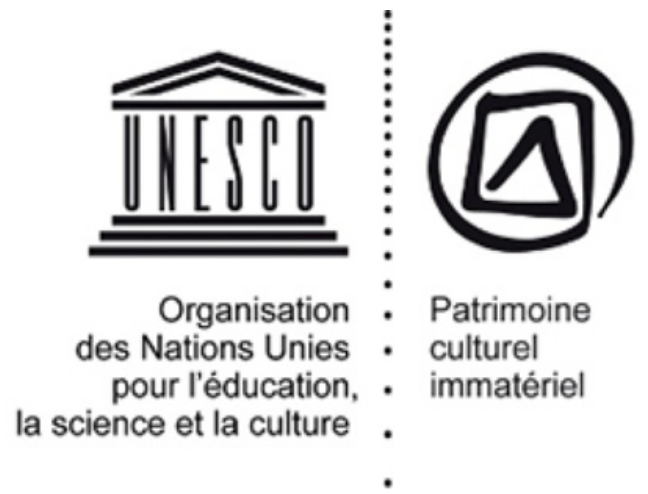

Figure 7. Logo du patrimoine culturel immatériel de l'UNESCO.

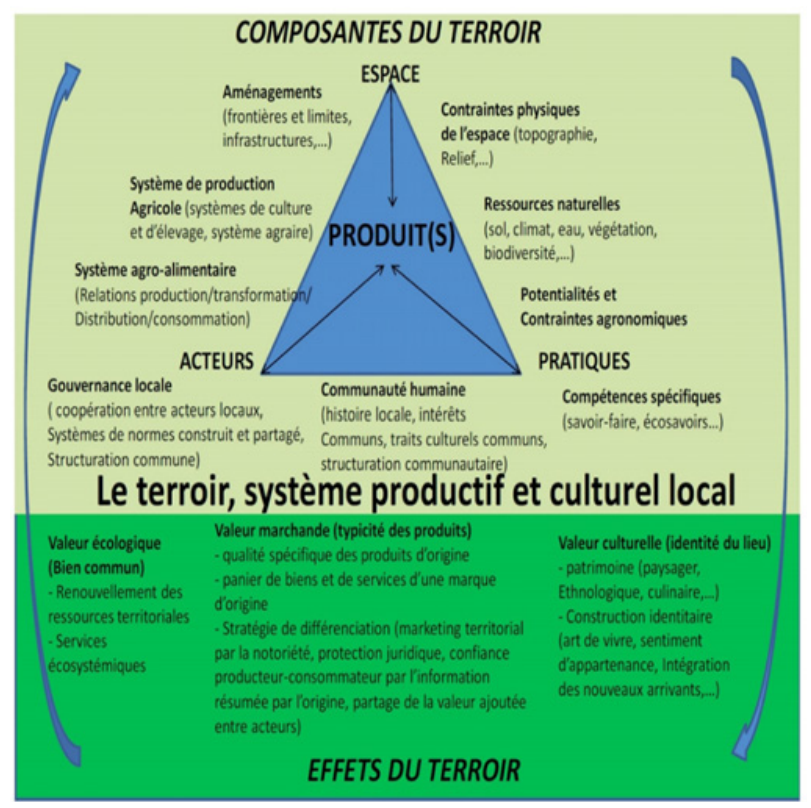

Figure 8. Composantes du terroir (source P. Prévost, C. Mathieu, F. Gautier-Pelissier et coll., 2014).

«L terroir exprime un patrimoine commun dans un processus de valorisation des ressources locales. Il offre la possibilité de porter, autour d'un (ou de plusieurs) produit(s) typique(s), des pratiques sociales, techniques et économiques procédant d'une vision et d'une vocation. En s'inscrivant dans une profondeur historique et en s'appuyant sur la construction par les acteurs de règles de normalisation et de gouvernance adaptées, le terroir est un système dynamique. Il peut constituer une voie de gestion durable des ressources et de développement adaptée à la communauté qui s'y rattache. Dans cette perspective, le terroir peut aussi être le motif d'un questionnement éthique renouvelé, spécialement à l'endroit de la négociation des règles de normalisation et de gouvernance.» Philippe Prévost, Mathieu Capitaine, François Gautier-Pelissier et coll., Le terroir, un concept pour l'action dans le développement des territoires, 2014 (Fig. 8).

Les monastères ont contribué à développer la vigne dans la plupart des régions européennes grâce notamment aux connaissances viticoles des moines sur l'art de cultiver la vigne (choix des plants, taille, terroir) et d'élaborer le vin. Historiquement la chute de l'empire romain

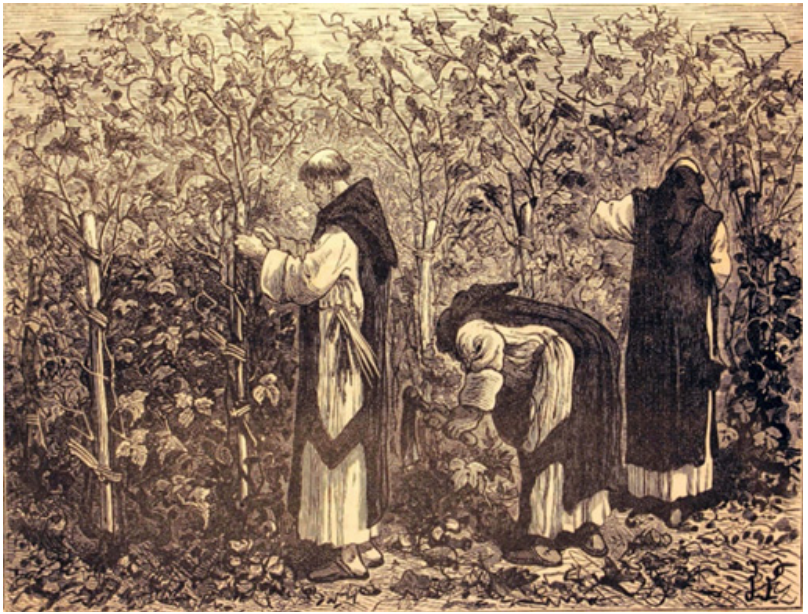

Figure 9. Les moines bourguignons cultivant la vigne, au moyen âge. Extrait de l'ouvrage de M. Figuier, Les merveilles de l'industrie, 1873.

fut suivie de plusieurs siècles de trouble et d'invasion pendant lesquels les dynasties franques des Mérovingiens (486-751) et des Carolingiens (751-987) ne réussit à imposer que de brèves périodes de calme, laissant l'église chrétienne s'affirmer comme le principal support de stabilité. Monastères et abbayes devinrent alors des centres d'érudition et des pôles artistiques tout en régissant de vastes domaines agricoles et viticoles.

«Nécessité culturelle et sociale, nécessité religieuse, nécessité économique, car le vin revendu fournit une part conséquente du fonctionnement financier des monastères, chaque abbaye en Europe devient donc un pôle viticole majeur. Les moines assurent aussi la transmission des livres agricoles des Romains, reprenant et améliorant ainsi les techniques culturales que les Anciens avaient mises au point. C'est aux moines que l'on doit l'amélioration du pressoir, la mise en valeur des meilleurs crus et terroirs, l'arrêt du complantage, l'édification de murets, pour dépierrer les sols et protéger les vignes de l'assaut du bétail. L'apport agronomique de ces hommes de Dieu est considérable.» Jean-Baptiste Noé http: //www.jbnoe.fr/.

Les climats du vignoble de Bourgogne, classés «Patrimoine Mondial» par l'Unesco, expriment avec raffinement le génie monastique du Moyen Âge. Sans disposer des techniques d'analyse moderne, les moines, dans l'ombre des monastères, avaient su, avec curiosité, intelligence, courage et passion, déterminer la relation entre la quintessence d'un vin et le micro-terroir qui en est à l'origine. Ainsi les parcelles bourguignonnes se distinguent les unes des autres par leurs conditions naturelles spécifiques (géologie, exposition, cépage...) qui ont été façonnées par le travail humain et peu à peu identifiées par rapport au vin qu'elles produisent. Ce paysage culturel est composé de plusieurs éléments: des parcelles viticoles et les unités de production associées, des villages et la ville de Beaune (Figs. 9 et 10).

Au-delà du zonage qualitatif empirique, les moines avaient une expertise de dégustateurs de raisin et de vin, une connaissance subtile des caractéristiques climatiques de l'année avec le recul des millésimes précédents, leur permettant ainsi de définir avec précision l'optimum de 


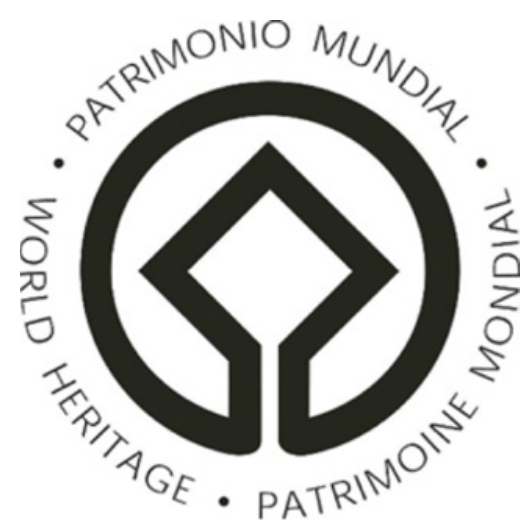

Figure 10. Les climats de Bourgogne figurent sur la liste du patrimoine mondial Unesco depuis 2015.

vendange Cette date historique de récolte est souvent un bon indicateur des particularités climatiques de l'année., Répertoriée avec rigueur par les moines, elles constituent une mémoire qui permet aux historiens de reconstituer le climat depuis plusieurs siècles.

Comme le précise V. Daux, le ban des vendanges constitue, «des archives continentales d'un grand intérêt du fait de leur résolution annuelle, de leur datation précise et des relations fortes et statistiquement significatives qui les lient à la température moyenne de la période de maturation du raisin».

Dans un ouvrage remarquable «Histoire du climat depuis l'an mil», Emmanuel Leroy Ladurie a retracé l'évolution des données climatiques à partir d'une série d'indicateurs, parmi lesquels les dates de vendange des régions septentrionales. Sa démarche s'appuyait sur des données historiques répertoriées dans différentes régions (Argenteuil, Bourgogne, Champagne, Jura, Suisse). Ces données étaient calculées à partir de la date des bans de vendange en nombre de jour à partir du $1^{\mathrm{er}}$ septembre. E. Leroy Ladurie a souligné la variabilité, au cours du temps des dates de récolte, en liaison avec des modifications climatiques.

Cette approche permet de reconstituer l'histoire climatique à partir d'une corrélation entre les dates de vendange et les conditions climatiques de la période végétative de la vigne. L'étude d'années atypiques telle 1816 (vendange très tardive) qui faisait suite à l'explosion du volcan Tambora en Indonésie, à l'origine d'un refroidissement significatif dans l'hémisphère nord souligne la pertinence de la démarche. Cette approche permet de conforter la probabilité d'un lien entre l'avancée progressive de la date de récolte depuis deux à trois décennies et des changements climatiques probablement à relier à des effets anthropiques. D'un point de vue pratique cette étude historique, souligne qu'une élévation de $1^{\circ} \mathrm{C}$ correspond en moyenne à une avancée d'environ 10 jours des dates de vendanges.

«Ce serait cependant trop restreindre la portée $d u$ ban des vendanges, que de lui attribuer uniquement la facilité de percevoir des redevances. Il avait aussi pour dessein plus noble, la recherche de la qualité, car les «experts» envoyés dans les vignes pour fixer la date du ban, avaient pour mission essentielle de s'assurer que les raisins avaient atteint la parfaite maturité à l'ouverture $d u$ ban.» Pierre Vital, Les vieilles vignes de notre France, 1956.

\subsection{Valorisation du savoir-faire de l'élaboration en amphores : exemple géorgien de l'élaboration en kvevris}

Depuis quelques années des amphores ou jarres, connaissent un certain développement dans les chais. Celles-ci, en terre cuite, en béton ou céramique, sont valorisées par des propriétés spécifiques concernant notamment la microoxygénation. Comme le souligne C. Caillaud, «À partir du Néolithique, l'usage de la céramique pour la réception du moût, sa vinification, son stockage et son transport semble se généraliser dans les civilisations orientales et méditerranéennes en même temps que se répand la vitiviniculture et la consommation du vin, comme l'attestent les très nombreux témoignages archéologiques. (... Les contenants en céramique, dolia et amphores, vont subir la concurrence des contenants en bois en Italie, peutêtre dès le Ier s. ap. J.-C., d'abord modestement puis de plus en plus fortement à partir des IIe-IIIe s. ap. J.-C., même si l'on connaît quelques productions d'amphores italiennes datant du IVe-Ve s. ap. J.-C. Il semble, en tout cas, qu'à la fin de l'Antiquité, l'usage des contenants céramiques disparaît de manière quasi définitive de la péninsule italique, en attendant que des vignerons éclairés redécouvrent les bienfaits des jarres en terre cuite.»

La Géorgie, berceau de la viticulture, a su maintenir cette production traditionnelle notamment au sein des domaines familiaux. La méthode géorgienne de vinification à l'ancienne dans des kvevris a été inscrite en 2013 sur la liste représentative du patrimoine culturel immatériel de l'humanité. La vinification en «kvevri» type de jarre (de l'arabe «djara» signifiant vase d'argile a large bouche) se pratique toujours dans les communautés rurale. L'élaboration traditionnelle consiste à fouler les raisins puis à les transférer dans un Kvevri scellé et enfoui dans le sol afin de laisser le mélange fermenter pendant cinq à six mois avant sa consommation. L'Unesco précise: «L vin joue un rôle important dans la vie quotidienne des Géorgiens et dans la célébration des rituels et des événements laïques ou religieux. La cave à vin est encore considérée comme le lieu le plus sacré du foyer. La tradition de la vinification en kvevri définit le mode de vie des communautés locales et constitue une part indissociable de leur identité culturelle et de leur héritage, les vignes et le vin étant évoqués dons les traditions orales et les chansons géorgiennes» (Fig. 11).

A partir de cette élaboration traditionnelle, des études ont été menées pour comprendre les mécanismes physiques en fonction de la forme et du type de récipient, notamment liés à la circulation du vin à l'intérieur de ces contenants comparativement à des cuves classiques. Ces études ont pu montrer l'intérêt de cette configuration traditionnelle en particulier vis-à-vis du processus de clarification naturelle.

\subsection{De la tradition écologie d'une cave : exemple de la cave bioclimatique du domaine Léon Boesch en Alsace}

La cave est un lieu de calme et de mystère où s'épanouit le vin, en quête de fraîcheur, d'obscurité. Ces paysages souterrains traditionnels témoignent d'un labeur au quotidien, d'un savoir-faire ancestral, d'une géologie singulière et parfois d'une histoire des architectures de surface, bâties avec les matériaux qui sont extraits des 


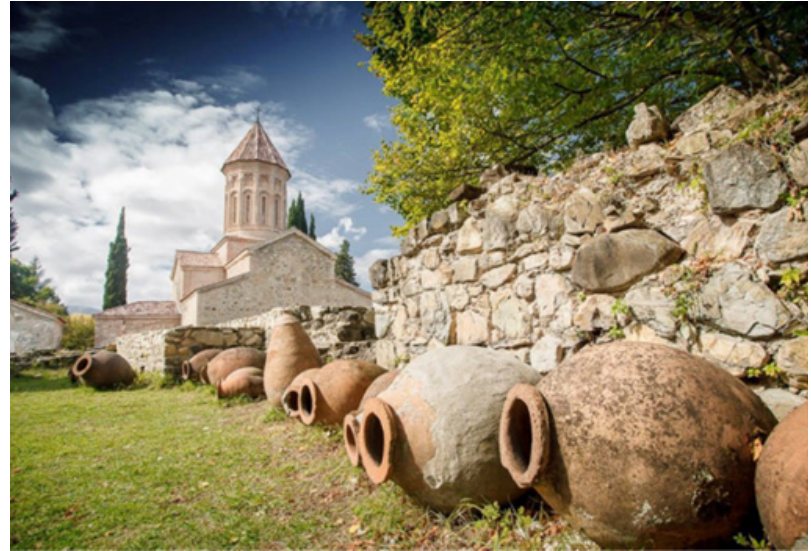

Figure 11. Photos de Géorgie qui souligne par les jarres (Kveris) au premier plan la tradition viticole ancestrale, associée à la religion orthodoxe (Source Fotolia).
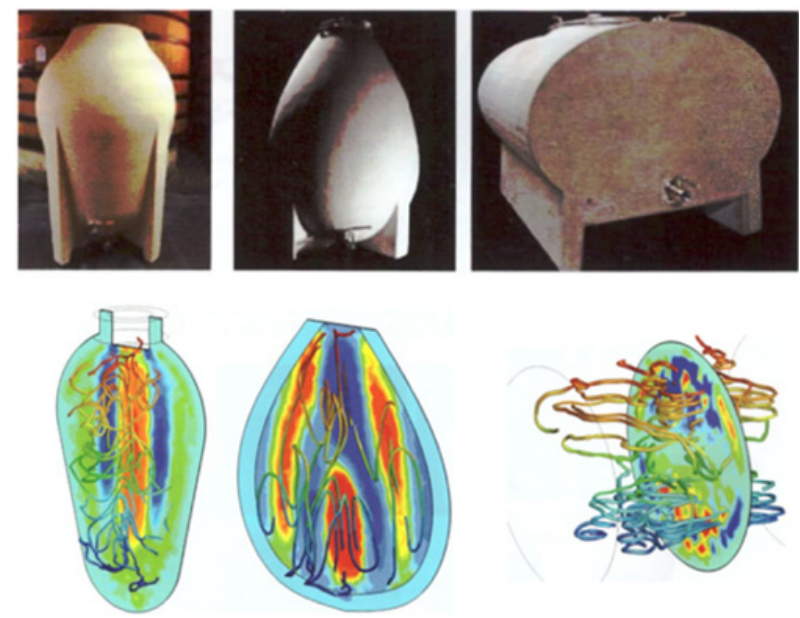

Figure 12. Simulation numérique par le modèle Aquilon des cuves, la vitesse verticale est représentée dans le sens de la gravité (en rouge : positive, en bleu : négative) (Source : R. Guillaument, J.P. Caltagirone, Revue française d'œnologie, 2016).

profondeurs. Les caves ou chais regroupent selon les régions des locaux aux fonctions multiples: local de pressurage, cellier de fermentation et de conservation des vins, cave de conservation des fûts ou des bouteilles, bâtiment d'embouteillage et de stockage.

La conception des chais traditionnels associe tout à la fois une maîtrise naturelle de la température et de l'hygrométrie et un transfert optimal de la vendange des vins et des bouteilles. Ainsi autrefois, le vigneron privilégiait la gravité avec en surface les locaux consacrés au pressurage et à l'embouteillage, les chais de vinification partiellement ou totalement enterrés et la cave en profondeur (Fig. 13).

La modernité a permis de s'affranchir de ces contraintes naturelles grâce à la climatisation, aux pompes et aux dispositifs de transfert du marc, des moûts et des vins mais avec une dépense énergétique croissante, et très souvent un impact sur l'effet de serre ainsi qu'une augmentation de la consommation d'eau qui doit être optimisée notamment dans la perspective de changement climatique. Les nouvelles conceptions écologiques s'inspirent des configurations traditionnelles de nos caves, qui utilisent notamment l'effet d'inertie

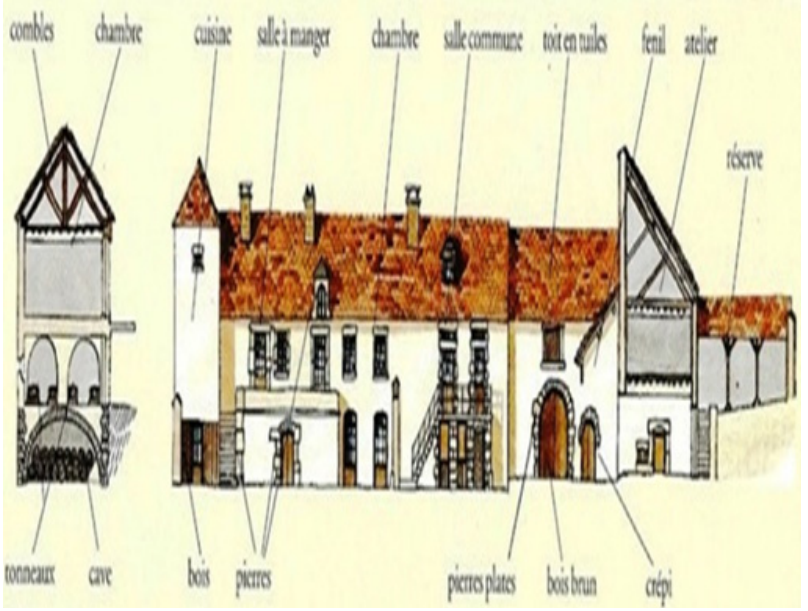

Figure 13. Maison vigneronne traditionnelle de la Côte-d'Or. La conception, qui valorisait l'inertie thermique du sous-sol, le choix de l'exposition permettait d'obtenir une parfaite articulation entre les contraintes de la zone d'habitation et de production des vins vis-à-vis notamment de la régulation température (Source : Encyclopédie touristique des vins de France, édition Hachette, 1994).

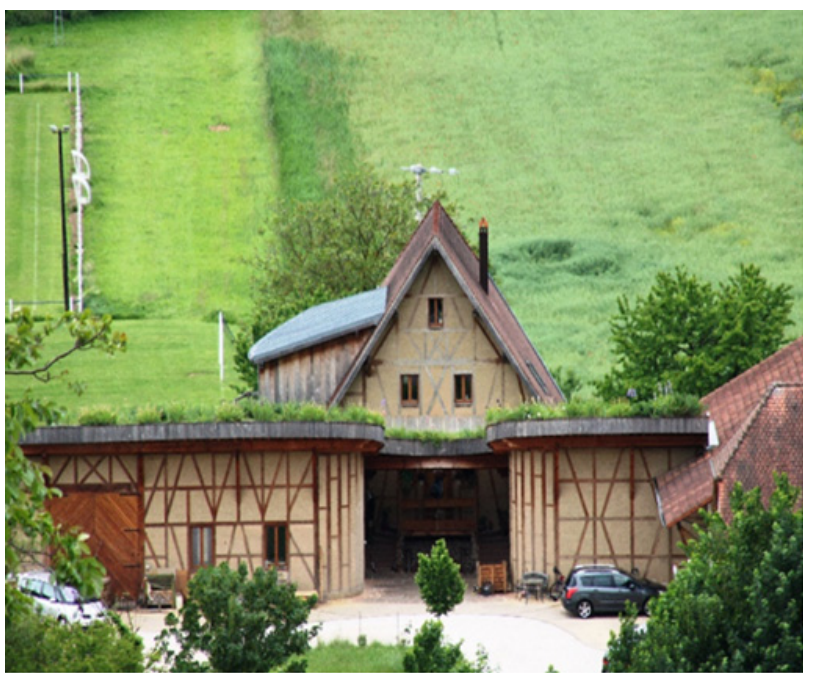

Figure 14. Domaine Léon Boesch en Alsace : exemple de conception d'une nouvelle cave bioclimatique qui s'inspire de l'architecture traditionnelle.

thermique du sous-sol, la végétalisation et une disposition optimale en fonction de l'exposition. Parallèlement, de nouvelles technologies ou conceptions telles que la géothermie, le solaire, les toits et murs végétaux, ainsi que des dispositifs de traitement des effluents écologiques, s'intègrent progressivement dans l'architecture et la conception des nouvelles caves.

Conçu par l'architecte Matthieu Winter, le domaine viticole alsacien Léon Boesch est construit à partir d'une ossature bois, mode de construction traditionnel en Alsace. La construction implique notamment des fondations en pierres sèches, une toiture végétalisée, une isolation des murs en bottes de paille, etc. Les matières premières viennent des alentours du village viticole. Les fondations sont constituées de roches calcaires d'un mètre d'épaisseur permettant de garantir l'hygrométrie et la «respiration» de la cave (Fig. 14). 


\section{Conclusion}

Les savoirs faire viticoles constituent, au-delà des atouts locaux, un patrimoine humain universel irremplaçable. Le génie et le courage les hommes ont permis à de nombreux écosystèmes terrestres, y compris parfois les plus extrêmes, d'être valorisés afin d'exprimer la quintessence du vin, expression des terroirs, en lien avec les particularités géologiques, climatiques, historiques et culturelles locales.

Très tôt les échanges entre les peuples ont permis une confrontation des savoirs et ont contribué à dynamiser la créativité locale. En Eurasie, grâce notamment aux civilisations égyptiennes, helléniques et romaines, relayées au moyen âge par les monastères, chaque région viticole a su trouver des formes singulières de culture de la vigne et d'élaboration des vins, adaptées au contexte physique et historique local. Ce savoir-faire s'est transmis par la migration des hommes et des idées au pays du Nouveau Monde qui a su avec des idées nouvelles, développer une viticulture à la fois moderne mais également fortement imprégnée des savoir-faire ancestraux.

Au-delà des enjeux patrimoniaux, ces savoirs sont souvent porteurs d'une identité locale, de nature à fédérer les énergies collectives autour de valeurs culturelles. La fête des vignerons de Vevey, classée par 1'UNESCO patrimoine mondial immatériel, témoigne de cette dynamique, support d'une gouvernance, indispensable à la valorisation qualitative des vins, mais également au développement opérationnel de projets durables. Au-delà de la dimension territoriale, les savoir-faire viticoles sont également porteurs de dynamique locale, de préservation et de valorisation. À titre d'exemple, la conception traditionnelle des caves, qui s'appuie notamment sur l'inertie thermique du sous-sol, alimente la vision écologique de leur conception. Il en est de même de l'élaboration traditionnelle en jarres de terre cuite, avec notamment l'exemple des KVEVRIS classés en Géorgie au titre du patrimoine immatériel de l'UNESCO. Ce savoir-faire ancestral sert de support à la conception de cuves ovoïdes qui comportent des propriétés thermiques et de circulation interne du vin spécifiques par rapport aux contenants traditionnels. Parfois ces pratiques ancestrales, à l'image des dates du ban des vendanges, répertoriées notamment par les moines, permettent d'alimenter la réflexion, sur l'évolution du climat passé, pour mieux appréhender son évolution dans un contexte de changements climatiques. Le classement immatériel des climats de Bourgogne, intègre également cette aptitude des moines à déterminer et à répertorier avec précision l'optimum de la date de vendange.

«Après un temps de confiance irrationnelle dans le progrès et dans la capacité humaine, une partie de la société est en train d'entrer dans une phase de plus grande prise de conscience. (...) Le changement climatique est un problème global aux graves répercussions environnementales, sociales, économiques, distributives ainsi que politiques, et constitue l'un des principaux défis actuels pour l'humanité. Les pires conséquences retomberont probablement au cours des prochaines décennies sur les pays en développement.» Encycliques Pape François, 5 juillet 2013.

Au final, les savoirs historiques locaux contribuent à une culture locale et une gouvernance, indispensable à la valorisation des terroirs et des vins, mais également au développement de démarches opérationnelles durable en lien notamment avec une responsabilité sociale et environnementale de la filière viticole, vis-à-vis des territoires mais également de notre planète et des générations futures. Ainsi le secteur viticole, probablement plus que tout autre filière agricole, se doit d'intégrer ce capital historique que constituent les savoir-faire locaux dans une vision de Responsabilité Sociétale et Environnementale universaliste.

\section{Références}

[1] Ph. Prévost, M. Capitaine, F. Gautier-Pelissier et coll., Le terroir, un concept pour l'action dans le développement des territoires, Vertigo revue électronique en sciences de l'environnement $\mathbf{1 4}$ Numéro 1, mai, www . vertigo.revues .org (2014)

[2] R. Guillaument, J.P. Caltagirone, Comment définir la cuve la mieux adaptée à ses besoins pour optimiser sa production et obtenir l'équilibre souhaité ? Revue française d'œnologie, Novembre-Décembre, N` 279 (2016)

[3] Ch. Caillaud, Le phénomène du «vin en amphore» dans l'Italie d'aujourd'hui, Territoires du vin $\mathrm{n}^{\circ} 6$ Territoires du vin d'Italie, 20 août, disponible sur Internet http://revuesshs.u-bourgogne.fr/ territoiresduvin/document.php?id=1880 ISSN 1760-5296 (2014)

[4] E. Joseph Stieglitz, Le prix de l'inégalité, Editions Les Liens qui Libèrent (2012)

[5] A. Touraine, Critique de la modernité, Éditions Fayard (1992)

[6] J.B. Noé, Les moines, la vigne, le vin, www . jbnoe. $\mathrm{fr} /$ Les-moines-la-vigne-le-vin

[7] V. Daux, La reconstruction climatique à partir des dates de vendanges, Revue de la BNF, 3(36), 26-33 (2010)

[8] E. Leroy-Ladurie, Histoire du climat depuis l'an mil, éditions Champs Flammarion (1983)

[9] P. Vital, Les vieilles vignes de notre France, réédition Wallada (1984) 